\title{
A review on contemporary practices in Lithography
}

\author{
Perna Kishor Krishna, Mantha Anil Srimanth, P.Sairaghava Reddy, \\ M.TECH, Department of ECE, Sreenidhi Institute of Science and Technology \\ M. TECH, Department of ECE, Sreenidhi Institute of Science and Technology \\ M.TECH, Department of ECE, Sreenidhi Institute of Science and Technology
}

\begin{abstract}
This paper discusses the basic concepts and current state of development of LITHOGRAPHY i.e., EUV lithography (EUVL), lithography that uses extreme ultraviolet (EUV) radiation with a different wavelengths in the range of 32 to 22 nanometers $(\mathrm{nm})$ to carry out projection imaging. Currently, and for the last several decades, optical projection lithography has been the lithographic technique used in the high volume manufacture of integrated circuits. It is widely anticipated that improvements in this technology will allow it to remain in semiconductor industry's workhorse through the $100 \mathrm{~nm}$ generation of devices. However, sometime around the year 2005, so-called NGL is used. EUVL is one such technology vying to become the successor to optical lithography. This paper provides an overview of the capabilities of EUVL, and explains how EUVL might be implemented.
\end{abstract}

Key words: Lithography, EUVL, Photo lithography, NGL.

\section{Introduction}

In the semiconductor industry Lithography is the most challenging technology. It is introduced to the semiconductor industry in 1958, when ICS were introduced. It is a patterning method that creates an IC layout on a resist layer of a silicon wafer or other semiconducting substrates. It mainly consists of three parts: a) The pattern printer b) Photo resist technology and c) The mask fabrication. The lithography which used in the firstly was light of the visible g-line $(436 \mathrm{~nm})$ and the ultraviolet i-line $(365 \mathrm{~nm})$. With the technology progress and the reduction of the size, the wavelength of the exposure light had to be reduced many times. When the IC feature size was reduced to $500 \mathrm{~nm}$ i.e., half a micron, the g-line \& i-line could no longer be used, then deep ultraviolet $248 \mathrm{~nm} \mathrm{KrF}$ and $193 \mathrm{~nm} \mathrm{ArF}$ excimer lasers were introduced into the semiconductor industry. The next generation lithography (NGL) is known as shorter wavelength lithography is studied to produce the smaller featured Ic's and so on.NGLuses shorter UV light $(157 \mathrm{~nm})$, EUV light (e.g. $13.5 \mathrm{~nm})$, X-ray $(0.4 \mathrm{~nm})$, and the even shorter wavelengths of electron and ion beams.

Detailed description of steps involved in Lithography: Coating of resist. Involves Spin coating and soft baking. Uses a wafer with an oxide layer on top.1. Oxide formation by heating the surface $900-1150^{\circ} \mathrm{C}$ in steam or humidified oxygen stream, oxide serves as a Mask for subsequent wet etching (Oxidation furnace).

2. Photo resist (usually an organic polymer) sensitive to UV is deposited; wafer is kept on a spinner (a vacuum Chuck holds the wafer in place, spinning at 1500-8000 rpm depending on the Viscosity of the resist. Required thickness. Film thickness uniformity of $\sim 5 \mathrm{~nm}$ is required for $(1.5 \mu \mathrm{m}$ film $)$ within and between layers.

For IC's resist thickness: $0.5-2 \mu \mathrm{m}$ Greater thickness required for 3D structures. Thickness optimization by Resist disperse rate. Dispense volume. Spin speed. Ambient temperature and humidity.

3. After spin coating, resist contains up to $15 \%$ organic solvent. This is removed by soft baking at $75-100^{\circ} \mathrm{C}$ for Approximately 10 mins. This step also Releases stress Improves adhesion of resist to wafer Process optimization by: Temperature profile Time/duration.

4. After soft baking, the resist coated wafers are transferred to an exposure system when they are aligned with the Features on the mask. Exposure and post-exposure treatment a very good alignment is required. Usually an UV lamp is used to illuminate the resist Proper intensity Directionality Spectral characteristics Uniformity across the wafer Means of Exposure.

UV: Extreme UV (EUV) $10-14 \mathrm{~nm}$

Deep UV (DUV) $150-300 \mathrm{~nm}$

Near UV (UV) 350-500nm

Typical:

G-line: $436 \mathrm{~nm}$ \}

H-line: $405 \mathrm{~nm}$ \} of a high intensity mercury lamp

I-line: $365 \mathrm{~nm}$ \}

Note: smallest feature size by projection lithography is the same as the $\lambda$ of the UV source.

When UV is used, typical resolution: $\sim 1 \mu \mathrm{m}$, registration $\sim 0.5 \mu \mathrm{m}$, throughput: $50-100 \mathrm{Wafers} / \mathrm{hr}$.

Post exposure treatments: 
- Post exposure baking.

- Flood exposure with other types of radiation.

- Treatment with reactive gas.

- Vacuum treatment.

5. Development transforms latent resist images joined during exposure into a relief image that will serve as a Mask during the subsequent processing. (Development)

- Selective dissolving of resist.

- Wet development (by immersion or spray-for a fixed period in a developer bath) by solvents, makes use of Variation in molecular weight. Polarity changes. (Occurring while exposure) Reactivity changes

6. De-scrumming and post-baking Descumming by a mild oxygen plasma treatment to remove unwanted resist left behind after development. Patterned resistances are also thinned during this process. Post-baking of wafer before subsequent steps (etching/deposition) to remove residual solvents. Annealing of film to improve adhesion Improves hardness of the film. Post -baking is also called hard-baking, done at $120^{\circ} \mathrm{C}$ for approximately 20 minutes.

7. Etching of the oxide layer

8. Resist stripping

Wet stripping - complete removal of the photo resist without damaging the device under construction, Use strong acid $\mathrm{H} 2 \mathrm{SO} 4$ or acid -oxidant combination: $\mathrm{H} 2 \mathrm{SO} 4-\mathrm{Cr} 2 \mathrm{O} 3$ attack the resist not the oxide/ $\mathrm{Si}$, Also in use: organic solvent strippers. e.g.: acetone.

Dry stripping (ashing) fewer disposal problems. More controllable. Chemicals used do not disintegrate with time. Doesn't cause undercutting/ broadening of photo resist features?e.g.: Reactive plasma stripping with oxygen gaseous chemical reactants (ozone) radiation (UV).

\section{Theoretical limits of photo lithography:}

Factors affecting resolution: Diffraction of light at the edge of an opaque feature in the mask as the light passes through alignment of wafer to mask, non-uniformities in wafer flatness, debris between mask and wafer.For $\lambda=400 \mathrm{~nm}, z=1 \mu \mathrm{m}$, Resolution is approximately $1 \mu \mathrm{m}$.

\section{Types of Lithography:}

Micro lithography which is features of smaller than $10 \mathrm{~mm}$ and Nano lithography which features of smaller than $100 \mathrm{~nm}$. And the other Lithography techniques are

A. Photolithography (optical, UV, EUV)

B. E-beam/ion-beam lithography

C. X-ray lithography

D. Nan imprint

E. Interference lithography.

\section{Extremeultra Violet Lithography}

Mostly other NGLs use one-fold image reduction membrane masks; EUVL uses masks with four-fold image reduction, which makes mask fabrication easy with the current technology. However, in closing the 157 $\mathrm{nm}$ lithography, the industry has created a technological long jump from $193 \mathrm{~nm}$ to $13.5 \mathrm{~nm}$ wavelength, creating complex challenges across the board. Therefore, EUVL technology includes EUV resist technology, EUV aligners or printers, and EUV masks, as well as metrology, inspection, and defectivity controls and many things.

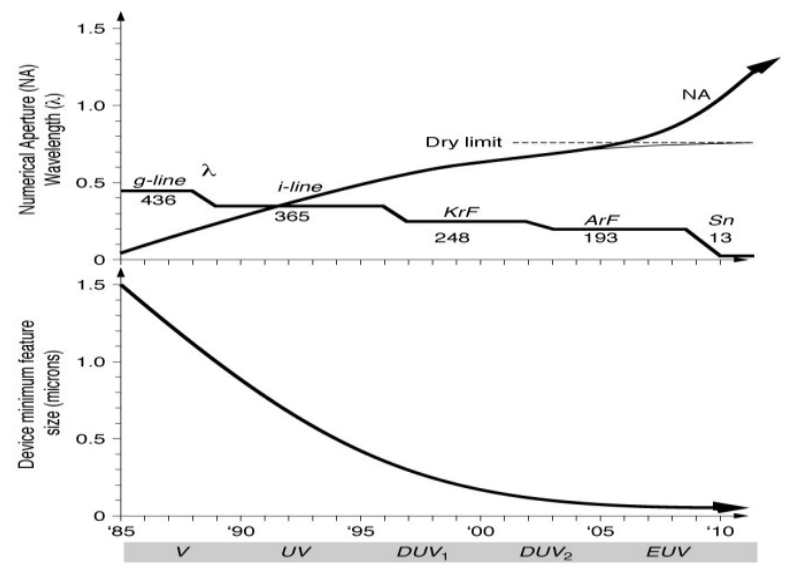


The most delayed lithography is EUVL implementation; optical lithography, on the other hand, has its inherent limit in resolution (R) and depth of focus (DOF), as shown by the following two equations:

$$
\begin{aligned}
& \mathrm{R}=\mathrm{k} 1 \lambda / \mathrm{NA} \\
& \mathrm{DOF}=\mathrm{K} 2 \lambda / \mathrm{NA} 2
\end{aligned}
$$

Where $\lambda$ is the wavelength, A the numerical aperture, and where $\mathrm{k} 1$ and $\mathrm{K} 2$ are constants.

Introduction:Conventional optical lithography has continuously improved the resolution by stretching all the factors of the equation determining the smallest printable feature size.

Smallest feature $=k 1 \lambda / N A$

The numerical aperture has been increased; the $k 1$-factor and the wavelength have been decreased. However, there will eventually be a limit to how far these factors can be stretched. The wavelength reaches its lower limit when all materials become highly absorbing and opaque, making manufacturing of refractive lenses impossible. A natural step is then to switch to reflective optics, but the problem is, as will be discussed below, that a reduced freedom of optical design will limit the achievable NA. However if the reduction in wavelength is greater than the loss in NA, there is still a gain in resolution according to Eq. Thanks to the development of multilayer mirrors, sufficient reflectivities can be obtained at an order of magnitude shorter wavelength than DUV wavelengths, i.e., EUV wavelengths, making EUVLPossible. Before discussing EUVL in more detail, a brief introduction of these mirrors is therefore appropriate.

\section{Main challenges for EUV lithography:}

A EUVL stepper is a very complicated system and several technologies have to be advanced significantly in order for the technology to become an industrial reality. The following is a list of critical issues that were identified and prioritized by the steering committee of the 1st international EUV symposium in Dallas, October 2002. Most of the issues in the list will be covered in the following review of the components of a EUVL stepper.

1. Source output

2. Defect-free multilayer coated mask blank manufacturing, including inspection

3. Source \& condenser optics reliability

4. Cost of ownership of EUV lithography

5.Defect-free pattern mask manufacturing/commercial availability

6. Reticle defect protection (from inspection through exposure)

7. Effective contamination control of optical path (lifetime)

8. High NA optics manufacturing

9. Thermal management of reticle \& projection optics at high throughput

10. Resist - high sensitivity at low power with low line-edge roughness

The wafer level - the resist: The wafer levels include the scanning stage, the wafer and the resist. As for the scanning stage the main difference from conventional lithography is that the stage has to operate in vacuum. This is not anticipated to be a problem. As for the resist, on the other hand, several issues have to be solved. However, not all issues are related to EUVL specifically, but rather to the fact that EUVL might not be inserted until the $32 \mathrm{~nm}$ node, posing tough requirements on resolution and line width roughness (LWR) etc. . . . Specific problems for EUV resists are that they must have very low out gassing, also when illuminated by EUV and out-of-band radiation, not to contaminate the optics. Furthermore, reasonable transmittance of EUV radiation is required to obtain good resist side-wall profiles. Two issues of the resists are related strongly to the source. First, the power requirement for the source is inversely proportional to the resist sensitivity. The current goal for the resist sensitivity is $2-5 \mathrm{~mJ} / \mathrm{cm} 2$, where certainly the lower number is preferable from the source point of view. Unfortunately studies indicate that the LWR values increase for resists with higher sensitivities. Second, the response of the resist for non-EUV radiation will influence the out-of band requirements of the source. In particular, DUV radiation may expose the resist. Actually, many EUV resists are based on resists for $248 \mathrm{~nm}$ lithography, and are therefore especially sensitive at that wavelength.

The projection optics: The role of the projection optics is to demagnifying and transfer the image of the mask to the resist-coated wafer. It has to resolve the smallest features of the chip design with high contrast over a large field. In conventional DUV objectives, a great number of lenses can be used to obtain the desired performance as illustrated however, in an all-reflective design only few mirrors can be used due to two main reasons. First, the effect of obscuration, i.e., one mirror cannot obscure the beam-path between other mirrors, making the design with a great number of mirrors very difficult. Second, the reflectivity of each mirror is limited to $\gg 70 \%$, meaning that the use of many mirrors will drastically lower the total transmission of the optical system, requiring more power from the source. Since only few mirrors can be used, they instead require aspheric 
surfaces to achieve the imaging requirements. Illustrates one suggested optical design for EUVL projection optics. Six mirror systems will be needed in production scale steppers. Common for different designs of EUV projection optics is that aberrations are well corrected over a ring field centered on the optical axis as illustrated. This ring-field therefore has to be scanned over the mask pattern as illustrated.

The mask: As can be seen in the list of main challenges for EUVL, many of the items of concern are related to the mask. However, the mask will only be discussed briefly here since its performance is little related to the source. A review of requirements and potential solutions regarding EUVL masks is given in a paper by Hector .The EUVL mask is a multilayer substrate coated with a patterned absorbing Top layer. The general mask problem is that it must be manufactured and kept free from printable defects over its full area of »106£132 $\mathrm{mm} 2$. A difficult issue is the manufacturing of a defect-free multilayer substrate. Substrates with 0.05 added defects $/ \mathrm{cm} 2$ can be achieved, but another factor of 10 improvements is needed. A further problem, as discussed by Meiling et al. is to keep the mask defect free during operation and handling, especially since no pellicle 1 can be used due to that no material sufficiently transparent to EUV is available.

Contamination issues: Contamination of the optical path is one of the obstacles for EUVL to overcome since it will limit the lifetime of the optical system. Meiling et al. Talk about a lifetime target of 30,000 hours of exposures where the lifetime is defined as when an irreversible transmission loss of $\gg 10 \%$ is reached. From the source point of view, the problem is mainly connected to debris and ions destroying the first collector mirror. Here the other sources of contamination will briefly be mentioned. Two major issues of contamination exist in the optical path: oxidation and carbon growth on the mirror surfaces during EUV exposures. The mirror surfaces emit secondary electrons under EUV irradiation, and these electrons are responsible for dissociation of hydrocarbons adsorbed to the mirror surface and similar for adsorbed water. Several experiments have been performed, both with EUV illumination and by simulating EUV exposure with an e-beam, all Indicating unacceptable contamination rates. Carbon contaminations seem, however, possible to remove through in-situ cleaning but oxidation appears to be an irreversible process. Several methods are proposed to limit contaminations. The obvious is certainly to improve the vacuum conditions, but this may complicate the mechanical design to an unacceptable level. Instead, other methods have to be used. Klebanoff have, e.g., shown how an ethanol background can stop oxidation. A promising approach is to introduce oxidation resistant capping layers to the multilayer mirrors. Both ruthenium and carbon capping layers have proven effective to limit the oxidation.

\section{Conclusion}

This paper is discussed about the Lithography technique .This is the most challenging technology in the semi conductor industry. The most promising next generation lithography is extreme ultra violet lithography (EUVL).EUVL was proposed long ago in 1988, but its implementation has been postponed several times. The semiconductor industry is now using EUVL in a pre-production phase, and EUVL is implementing in $32 \mathrm{~nm}$ and $22 \mathrm{~nm}$ technological nodes. The currently wavelength is expected to be $13.5 \mathrm{~nm}$. And also in the above report we analyze the different types of lithography techniques such like photo Lithography, NGL and many are discussed. The EUV Lithography technique has been discussed mainly here. In future the Lithography technology node is less than the $12 \mathrm{~nm}$ range.

\section{References}

[1]. Banqiu Wu and Ajay Kumar (May 2009). Extreme Ultraviolet Lithography. McGraw-Hill Professional, Inc. ISBN 0-07-154918-8.

[2]. Banqiu $\mathrm{Wu}$ and Ajay Kumar (2009). "Extreme Ultraviolet Lithography: Towards the Next Generation of Integrated Circuits". Optics \& Photonics Focus

[3]. ExtremeUltraviolet Lithography: Towards the Next Generation of Integrated Circuits Optics\& Photonics Focus Volume 7 Story 4 - 4/11/2009

[4]. Extreme Ultraviolet Lithography: Towards the Next Generation of Integrated Circuits, Focus Volume 7 Story 4 - 4/11/2009

[5]. www.britannica.com/bcom/eb/article

[6]. Deforest, W.S., Photo resist. McGraw Hill, 1975 page(s)1 and 47

[7]. Reichmanis, Ober, McDonald, Iwayanag, Nishikubo., Microelectronics Technology, American Chemical Society, 1995 Page(s) xi, $1-5$ and 20

[8]. Www.mitsuichemicals.com/cheminter/photoraw/

[9]. Middleman, Stanley Hochberg Arthur., Process Engineering Analysis in Semiconductor Fabrication., McGraw-Hill, Inc 1993 page(s) 285-290.

[10]. B. A. M. Hansson, L. Rymell, M. Berglund, and H. M. Hertz, "A Liquid- Xenon-Jet Laser-Plasma X-Ray and EUV Source", Microel. Engin. 53, 667-670 (2000).

[11]. O. Hemberg, B. A. M. Hansson, M. Berglund and H. M. Hertz, "Stability of droplet-target laser-plasma soft x-ray sources", J. Appl. Phys. 88, 5421-5425 (2000).

[12]. B. A. M. Hansson, L. Rymell, M. Berglund, O. Hemberg, E. Janin, J. Thoresen,and H. M. Hertz, "Liquid-Xenon-Jet Laser-Plasma Source for EUV Lithography”, PIE 4506, 1-8 (2001). 
[13]. B. A. M. Hansson, M. Berglund, O. Hemberg, and H. M. Hertz, "Stabilization of liquefied-inert-gas jets for laser-plasma generation", submitted to J. Appl. Phys.

[14]. B. A. M. Hansson, S. Mosesson, and H. M. Hertz, "Improved emission uniformity from a liquid-jet laser-plasma EUV source", submitted to Appl. Opt.

[15]. P. A. C. Jansson, B. A. M. Hansson, O. Hemberg, M. Otendal, A. Holmberg, J. de Groot, and H. M. Hertz, "Liquid-metal-jet laserplasma extreme ultraviolet generation", submitted to Appl. Phys. Lett.

[16]. B. A. M. Hansson, O. Hemberg, H. M. Hertz, M. Berglund, B. Jacobsson, E. Janin, S. Mosesson, L. Rymell, J. Thoresen, and M. Wilner, "Characterizationof a liquid-xenon-jet laser-plasma EUV source", submitted to Rev. Sci. Instrum. 